\title{
Schottky barrier formed by network of screw dislocations in $\mathrm{SrTiO}_{3}$
}

\author{
Xin Guo a) \\ Department of Materials Science and Engineering, University of Florida, P.O. Box 116400, Gainesville, \\ Florida 32611-6400 and Institut für Festkörperforschung, Forschungszentrum Jülich, \\ 52425 Jülich, Germany \\ Zaoli Zhang and Wilfried Sigle \\ Max-Planck-Institut für Metallforschung, Heisenbergstraße 3, 70569 Stuttgart, Germany \\ Eric Wachsman \\ Department of Materials Science and Engineering, University of Florida, P.O. Box 116400, Gainesville, \\ Florida 32611-6400 \\ Rainer Waser \\ Institut für Festkörperforschung, Forschungszentrum Jülich, 52425 Jülich, Germany
}

(Received 13 May 2005; accepted 30 August 2005; published online 13 October 2005)

A network of screw dislocations was artificially created in $\mathrm{SrTiO}_{3}$, and characterized by transmission electron microscopy (TEM). Slight expansion of the dislocation core was observed by TEM. The composition of the dislocation core was quantified by electron energy loss spectroscopy, which reveals a $13 \pm 5 \% \mathrm{Ti} / \mathrm{O}$ ratio increase but no $\mathrm{Sr} / \mathrm{Ti}$ ratio change in the core, demonstrating an oxygen deficiency or oxygen vacancy surplus in the dislocation core. The vacancy surplus was estimated to be about one vacancy every unit cell along the core. The dislocation core is positively charged; therefore, oxygen vacancies and holes are expelled from the dislocation network, forming a double Schottky barrier, which blocks charge carrier transports across the network. The Schottky barrier height at $T=823 \mathrm{~K}$ and $P_{\mathrm{O}_{2}}=2 \mathrm{~Pa}$ was determined to be $\sim 0.48 \mathrm{~V}$. (c) 2005 American Institute of Physics. [DOI: 10.1063/1.2112202]

Acceptor-doped $\mathrm{SrTiO}_{3}$ is a model material for studying point-defect physics and chemistry; the behavior of point defects in the bulk crystal is well understood. ${ }^{1-3}$ In oxidizing to a moderately reducing atmosphere, typically $10^{-5}$ to $10^{5} \mathrm{~Pa} \mathrm{O}_{2}$ at $600 \mathrm{~K}$, acceptor-doped $\mathrm{SrTiO}_{3}$ is a mixed conductor of oxygen vacancies and holes. Electrons are also present, however, with a bulk concentration several orders of magnitude lower than those of oxygen vacancies and holes. Dislocations are also observed in the $\mathrm{SrTiO}_{3}$ lattice. ${ }^{4-7}$ Dislocations can be introduced into the lattice by plastic deformation, ${ }^{4}$ and crystallization of amorphous phase. $^{7}$ Usually the dislocation density is higher $(\sim 6$ $\times 10^{9} \mathrm{~cm}^{-2}$ ) at the surface, and it declines to a constant level of $\sim 10^{8} \mathrm{~cm}^{-2}$ at about $20 \mu \mathrm{m}$ from the surface. ${ }^{5}$ It was supposed that a relatively high density of dislocations in a $5 \mu \mathrm{m}$ thick skin layer facilitated the charge transport in $\mathrm{SrTiO}_{3}$ crystals. ${ }^{6}$ Please note that even in a dilute solution, the concentrations of point defects are many orders of magnitude higher than the dislocation density. For example, in 2.15 $\times 10^{18} \mathrm{~cm}^{-3} \mathrm{Fe}$-doped $\mathrm{SrTiO}_{3}$, the oxygen vacancy concentration is $\sim 10^{18} \mathrm{~cm}^{-3}$ and the hole concentration $\sim 10^{13} \mathrm{~cm}^{-3}$ at $T=600 \mathrm{~K}$ and $P_{\mathrm{O}_{2}}=1 \mathrm{~Pa}^{8}$

The density of dislocations in $\mathrm{SrTiO}_{3}$ thin films and foils can be significantly decreased by annealing, ${ }^{5}$ since dislocations are not equilibrium defects. Similarly, the high-density dislocations in $\mathrm{BaTiO}_{3}$ thin films can be annihilated by annealing at $1273 \mathrm{~K}$ for $2 \mathrm{~h} .{ }^{9}$ However, much higher annealing temperatures and longer annealing periods are necessary to annihilate the dislocations in bulk materials (e.g., single crys-

\footnotetext{
a) Author to whom correspondence should be addressed; electronic mail: xinguo@ufl.edu,x.guo@fz-juelich.de
}

tals and ceramics), because long-distance diffusions of sluggish cations are involved in the dislocation annihilation in such cases.

In this work, a network of screw dislocations was artificially introduced into $\mathrm{SrTiO}_{3}$. Unlike isolated dislocation lines that do not significantly influence the defect structure, dislocation networks or arrays constitute severe perturbations of the defect structure. The purpose of this work is to investigate the interaction between point defects and the dislocation network.

A network of screw dislocations was created by diffusion bonding of the (100) surfaces, with a twist angle of $\sim 1^{\circ}$, of two undoped $\mathrm{SrTiO}_{3}$ crystal platelets (purchased from CrysTec, Berlin) in ultrahigh vacuum $\left(10^{-9} \mathrm{~Pa}\right)$. The surfaces to be bonded were polished, ultrasonically cleaned in propanone, and sputter cleaned in situ using $\mathrm{Ar}^{+}$ions of about $1.3 \mathrm{keV}$ energy. The diffusion bonding was conducted at $1723 \mathrm{~K}$ for $2.5 \mathrm{~h}$ under $1 \mathrm{MPa}$ load. After bonding, the $\mathrm{SrTiO}_{3}$ crystal was oxidized in air at $1473 \mathrm{~K}$ for $24 \mathrm{~h}$. Please note that annealing cannot eliminate the dislocations created this way. The exact twist angle was measured to be $0.7^{\circ}$ from Kikuchi patterns. The crystal size was $4 \times 3 \times 1 \mathrm{~mm}^{3}$, with the dislocation network being parallel to the $4 \times 3 \mathrm{~mm}^{2}$ surface and situated approximately in the middle of the crystal slab. The composition of the crystal was analyzed by inductively coupled plasma mass spectroscopy; the major impurities are $\mathrm{Ba}(406 \mathrm{ppm}), B$-site acceptors $\mathrm{Mg}(38 \mathrm{ppm})$ and $\mathrm{Zn}$ (9.0 ppm), and an $A$-site donor La $(2.6 \mathrm{ppm})$, the concentrations of the other elements are below $1 \mathrm{ppm}$. Therefore, the nominally undoped $\mathrm{SrTiO}_{3}$ crystal is actually doped with surplus acceptor impurities $\left(\sim 5 \times 10^{18} \mathrm{~cm}^{-3}\right)$.

The dislocation network was characterized by transmission electron microscopy (TEM) (model: Philips CM20, op- 


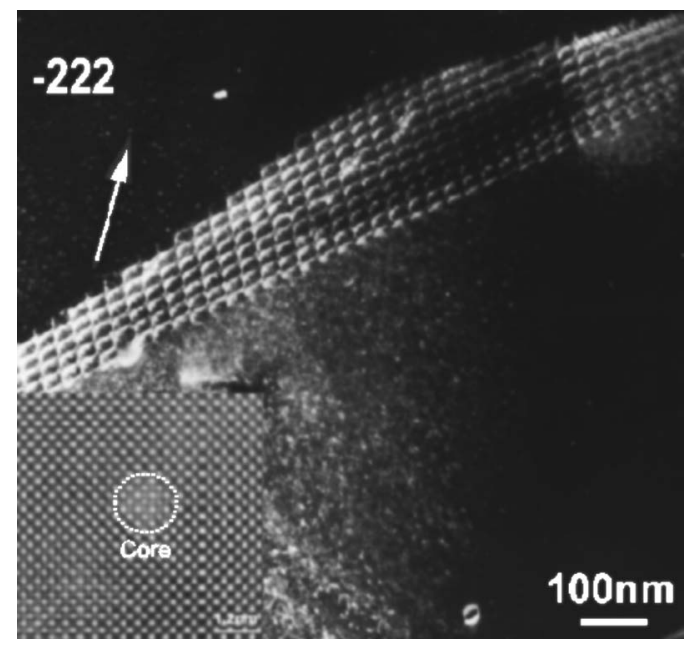

FIG. 1. Dark-field TEM image of screw dislocations. The inset shows a high-resolution TEM image of one of the dislocations viewed along the dislocation line. The core region, showing contrast different from the bulk, is encircled.

erated at $200 \mathrm{kV}$ with a point resolution of $0.27 \mathrm{~nm}$ ) and high-resolution transmission electron microscopy (model: JEOL ARM 1250, operated at $1250 \mathrm{kV}$ with a point resolution of $0.12 \mathrm{~nm}$ ). Figure 1 clearly shows the dislocation network using the diffraction vector $\overline{2} 22$. From the invisibility criterion $\mathbf{g} \cdot \mathbf{b}=0$, where $\mathbf{g}$ is the diffraction vector and $\mathbf{b}$ the Burgers vector, it was found that the dislocation network consists of two sets of $\langle 100\rangle$ screw dislocations, the diffraction vectors of which are 020 and $\overline{1} 01$. The average spacing between the dislocations was determined to be $31.2 \pm 0.8 \mathrm{~nm}$. The image of the dislocation core region is given in the Fig. 1 inset, which shows an almost perfect lattice. However, slight expansion of core region was observed. Moreover, some blurred contrast as well as some additional weak bright points are present as compared with the regular lattice, which can be accounted for by the core expansion. More structural details of the screw dislocation can be found in Ref. 10 .

$\mathrm{The} \mathrm{Sr} / \mathrm{Ti}$ and $\mathrm{Ti} / \mathrm{O}$ ratios of the dislocation core were quantified by electron energy loss spectroscopy (EELS) (model: Gatan UHV Enfina 766). The EELS quantification discloses that the $\mathrm{Ti} / \mathrm{O}$ ratio in the dislocation core is increased by about $13 \pm 5 \%$, while the $\mathrm{Sr} / \mathrm{Ti}$ ratio is almost identical to that of the bulk, demonstrating an oxygen deficiency in the core. In $\mathrm{SrTiO}_{3}$, Ti ions are surrounded by oxygen octahedra. Since adjacent octahedra share one corner, an oxygen ion always separates two Ti ions. The removal of an oxygen ion will inevitably lead to a repulsion of the two $\mathrm{Ti}$ ions, resulting in the observed core expansion. In other words, oxygen vacancy concentration in the dislocation core is higher than the bulk concentration; as a result, the dislocation core is positively charged. The oxygen vacancy surplus in the dislocation core has also been demonstrated for edge dislocations in $\mathrm{SrTiO}_{3}$ crystals. ${ }^{11,12}$

The electrical properties were investigated by impedance spectroscopy performed at an amplitude of $10 \mathrm{mV}$ (model: 1260 Frequency Response Analyzer, Solartron Instruments) within a temperature range of 573 to $823 \mathrm{~K}$ in $\operatorname{argon}\left(P_{\mathrm{O}_{2}}\right.$ $=2 \mathrm{~Pa})$. The measurement current direction was perpendicular to the dislocation network. Platinum electrodes were deposited on the $4 \times 3 \mathrm{~mm}^{2}$ surfaces by sputtering. As a reference, the impedance spectrum of a single crystal with same Downloaded 21 Dec 2006 to 134.94.122.39. Redistribution subject
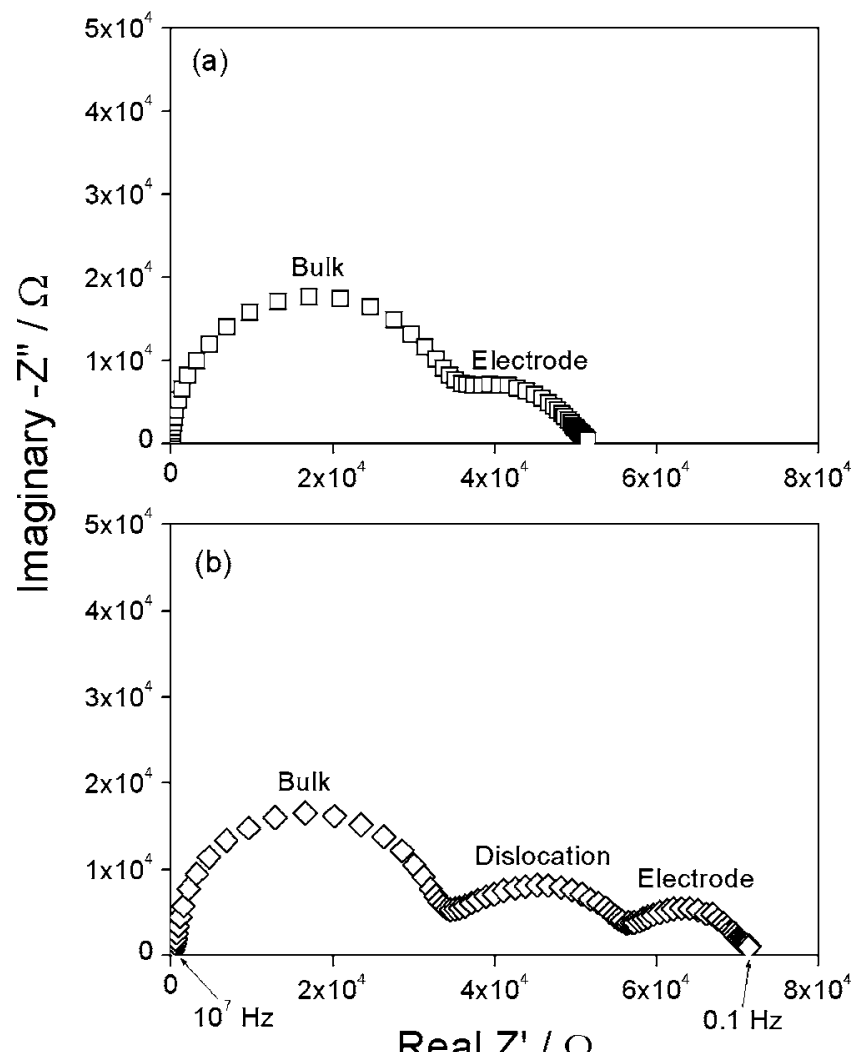

FIG. 2. Impedance spectra of (a) $\mathrm{SrTiO}_{3}$ single crystal and (b) $\mathrm{SrTiO}_{3}$ crystal with dislocation network measured at $773 \mathrm{~K}$ in argon.

composition and of similar dimension to the crystal with the dislocation network was first recorded. Within the frequency range of 0.1 to $10^{7} \mathrm{~Hz}$, two relaxation processes (semicircles) were recorded in the impedance spectrum of the single-crystal sample [Fig. 2(a)]. In the order of decreasing frequency, the two semicircles correspond to the impedance responses of the bulk and the electrode, respectively. However, three relaxation processes were recorded for the crystal with the dislocation network [Fig. 2(b)]. Similar to the single crystal, the high-frequency semicircle is due to the bulk, and low-frequency one due to the electrode. The extra relaxation process recorded for the crystal with the dislocation network in the intermediate frequency range [Fig. 2(b)] is due to the dislocation network. The impedance spectrum of the crystal with the dislocation network was fitted according to an equivalent circuit consisting of three $R Q$ ( $R$ : resistance, $Q$ : constant phase element) circuits in series. From the intersections of the fitted semicircles on the real axis, the bulk resistance and the resistance of the dislocation network were obtained. The bulk electrical conductivity is then simply $L /\left(R_{\text {bulk }} A\right)$. Here $R_{\text {bulk }}$ is the bulk resistance, $L$ is the sample thickness, and $A$ the cross-section area. The specific conductivity of the dislocation network was calculated from ${ }^{13}$ $\sigma_{\text {dis }}=C_{\text {bulk }} L /\left(C_{\mathrm{dis}} R_{\mathrm{dis}} A\right)$, where $R_{\mathrm{dis}}$ is the resistance of the dislocation network; $C_{\text {bulk }}$ and $C_{\text {dis }}$ are the capacitances of the bulk and the dislocation network, respectively. The capacitance values were obtained by fitting the impedance spectrum.

The electrical conductivities of the bulk and the dislocation network are plotted in Fig. 3 as a function of temperature. Within the temperature range of 573 to $823 \mathrm{~K}$, the specific conductivity of the dislocation network is about two to o AIP license or copyright, see http://apl.aip.org/apl/copyright.jsp 


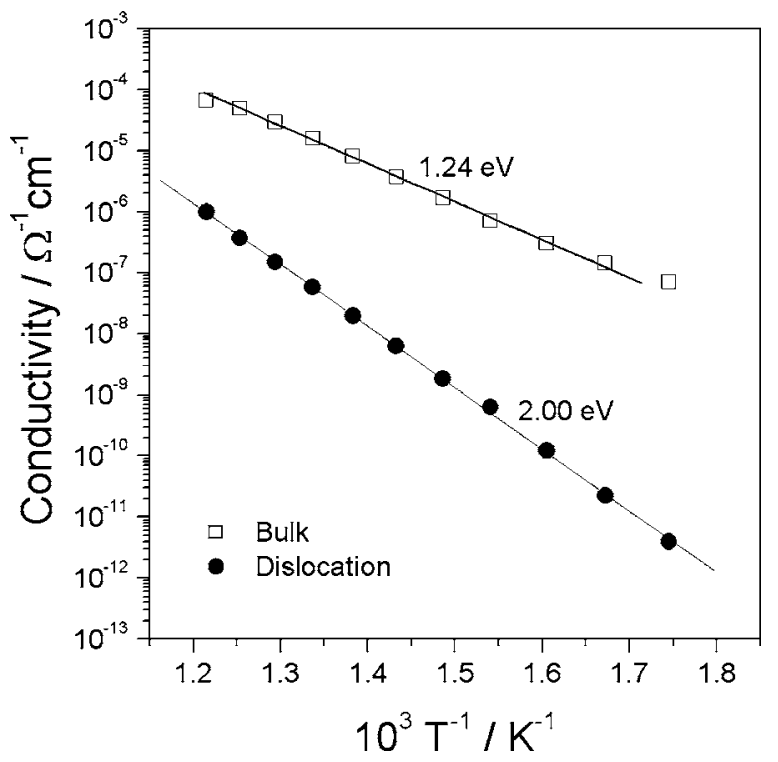

FIG. 3. Electrical conductivities of bulk and dislocation network.

three orders of magnitude lower than the bulk conductivity, and the activation energy is remarkably higher for the dislocation network. It is obvious that the dislocation network blocks the charge carrier (oxygen vacancy and hole) transport across it.

As already mentioned, the dislocation core is positively charged. The positive potential of the dislocation core expels oxygen vacancies and holes, forming depletion layers around the dislocations. Because of the different charge numbers (2 for oxygen vacancies and 1 for holes), the oxygen vacancy concentration, perceiving the same electric field, should decay much more steeply from the bulk toward the dislocation network than the hole concentration profile. Thus, a double Schottky barrier is formed across the dislocation network. At a high temperature, for example, $823 \mathrm{~K}$, both the electrical conduction in the bulk and across the dislocation network is predominantly electronic. Under this condition, the Schottky barrier height $\Delta \varphi(0)$ can be calculated from ${ }^{13,14}$

$$
\frac{\sigma_{\text {bulk }}}{\sigma_{\text {dis }}}=\frac{\exp \left[e \Delta \varphi(0) / k_{\mathrm{B}} T\right]}{2 e \Delta \varphi(0) / k_{\mathrm{B}} T} .
$$

At $T=823 \mathrm{~K}$ and $P_{\mathrm{O}_{2}}=2 \mathrm{~Pa}$, the Schottky barrier height of the dislocation network calculated from Eq. (1) is $\sim 0.48 \mathrm{~V}$. The width of the charge carrier depletion layer $\left(\lambda^{*}\right)$ can then be calculated from ${ }^{13,14}$

$$
\lambda^{*}=\left(\frac{2 \varepsilon \Delta \varphi(0)}{e c}\right)^{1 / 2},
$$

where $\varepsilon$ is the dielectric constant, $e$ the elementary charge and $c$ the dopant concentration. In deriving Eqs. (1) and (2), the frozen acceptor profile is assumed. At $T=823 \mathrm{~K}$ and $P_{\mathrm{O}_{2}}=2 \mathrm{~Pa}, \lambda^{*} \approx 42 \mathrm{~nm}$, which is larger than the distance between dislocations $(31.2 \mathrm{~nm})$. Therefore, the depletion layers of neighboring dislocations overlap; thus, the depletion layer of the dislocation network is continuous. Under this condition, the charge of the dislocation network per unit area $(Q)$ can be calculated from ${ }^{15,16}$

$$
Q=[8 \varepsilon c e \Delta \varphi(0)]^{1 / 2},
$$

which is $\sim 6.8 \times 10^{-6} \mathrm{C} \mathrm{cm}^{-2}$ at $T=823 \mathrm{~K}$ and $P_{\mathrm{O}_{2}}=2 \mathrm{~Pa}$, equivalent to a charge of $\sim+2 e$ per unit cell length of a dislocation core. This charge can be accounted for by one oxygen vacancy every unit cell along the dislocation core. It is noted that the core charge of screw dislocations is higher than that of edge dislocations $(\sim+0.93 e$ per unit cell length of a dislocation $\operatorname{core}^{15}$ ), this may be due to different core structures.

In conclusion, oxygen vacancy concentration in the core of the screw dislocation in $\mathrm{SrTiO}_{3}$ is higher than the bulk concentration; therefore, the dislocation core is positively charged. The positive potential of the dislocation core expels oxygen vacancies and holes, forming a double Schottky barrier. The Schottky barrier blocks the charge carrier transport across the dislocation network.

${ }^{1}$ N. H. Chan, R. K. Sharma, and D. M. Smyth, J. Electrochem. Soc. 128, $1762(1981)$.

${ }^{2}$ R. Waser, J. Am. Ceram. Soc. 74, 1934 (1991).

${ }^{3}$ F. Noll, W. Munch, I. Denk, and J. Maier, Solid State Ionics 86-88, 711 (1996).

${ }^{4}$ P. Gumbsch, S. Taeri-Baghbadrani, D. Brunner, W. Sigle, and M. Rühle, Phys. Rev. Lett. 87, 085505 (2001).

${ }^{5}$ R. Wang, Y. Zhu, and S. M. Shapiro, Phys. Rev. Lett. 80, 2370 (1998).

${ }^{6}$ K. Szot, W. Speier, R. Carius, U. Zastrow, and W. Beyer, Phys. Rev. Lett. 88, 075508 (2002).

${ }^{7}$ S. B. Lee, W. Sigle, F. Phillipp, and D. Brunner, Acta Mater. 53, 1843 (2005).

${ }^{8}$ X. Guo and J. Maier, Solid State Ionics 130, 267 (2000).

${ }^{9}$ H. P. Sun, X. Q. Pan, J. H. Haeni, and D. G. Schlom, Appl. Phys. Lett. 85, 1967 (2004).

${ }^{10}$ Z. Zhang, W. Sigle, and W. Kurtz, Phys. Rev. B 69, 144103 (2004).

${ }^{11}$ Z. Zhang, W. Sigle, and M. Rühle, Phys. Rev. B 66, 094108 (2002).

${ }^{12}$ Z. Zhang, W. Sigle, W. Kurtz, and M. Rühle, Phys. Rev. B 66, 214112 (2002).

${ }^{13}$ X. Guo and J. Maier, J. Electrochem. Soc. 148, E121 (2001).

${ }^{14}$ J. Fleig, S. Rodewald, and J. Maier, J. Appl. Phys. 87, 2372 (2000).

${ }^{15}$ R. A. De Souza, J. Fleig, J. Maier, O. Kienzle, Z. Zhang, W. Sigle, and M. Rühle, J. Am. Ceram. Soc. 86, 922 (2003).

${ }^{16}$ R. A. De Souza, J. Fleig, and J. Maier, J. Appl. Phys. 97, 053502 (2005). 